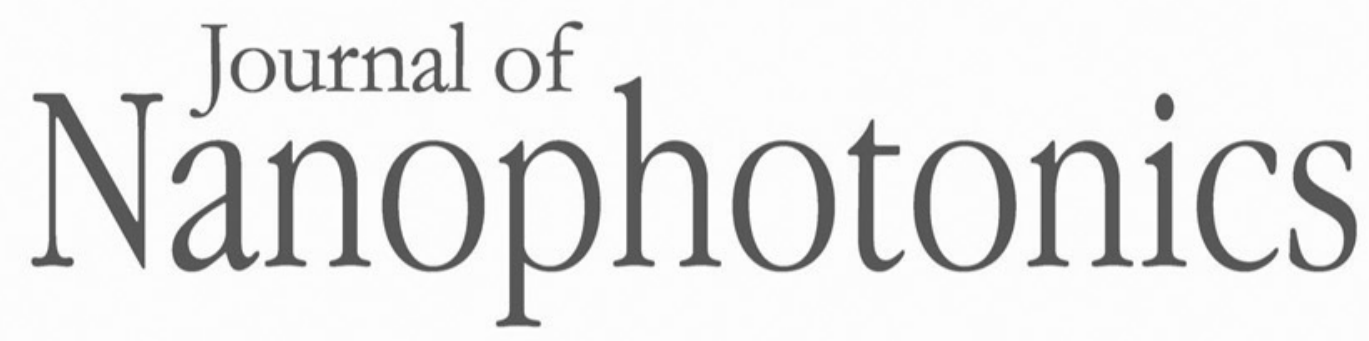

Nanophotonics.SPIEDigitalLibrary.org

\title{
Multiple trains of same-color surface plasmon-polaritons guided by the planar interface of a metal and a sculptured nematic thin film. Part V: Grating-coupled excitation (Erratum)
}

\author{
Muhammad Faryad \\ Akhlesh Lakhtakia
}




\section{Multiple trains of same-color surface plasmon- polaritons guided by the planar interface of a metal and a sculptured nematic thin film. Part V: Grating-coupled excitation (Erratum)}

\section{Muhammad Faryad $^{\mathrm{a}}$ and Akhlesh Lakhtakia ${ }^{\mathrm{b}, *}$}

${ }^{a}$ Lahore University of Management Sciences, Department of Physics, Lahore, Pakistan

${ }^{b}$ Pennsylvania State University, Department of Engineering Science and Mechanics, Nanoengineered Metamaterials Group (NanoMM), University Park, Pennsylvania, United States

[DOI: 10.1117/1.JNP.14.019901]

Equation (4) in this paper [J. Nanophoton. 5(1), 053527 (2011) [doi:10.1117/1.3663210] has a sign error:

$$
\underline{\underline{\epsilon}}_{g}(x, z)= \begin{cases}\epsilon_{m} \underline{\underline{\underline{I}}}-\left[\epsilon_{m} \underline{\underline{\underline{I}}}+\underline{\underline{\epsilon}}_{\mathrm{SNTF}}(z)\right] \mathcal{U}\left[d_{2}-z-g(x)\right], & x \in\left(0, L_{1}\right) \\ \underline{\underline{\epsilon N T F}}_{\mathrm{SN}}(z), & x \in\left(L_{1}, L\right)\end{cases}
$$

The correct Eq. (4) is as follows:

$$
\underline{\underline{\epsilon}}_{g}(x, z)= \begin{cases}\epsilon_{m} \underline{\underline{I}}-\left[\epsilon_{m} \underline{\underline{I}}-\underline{\underline{\epsilon}}_{\mathrm{SNTF}}(z)\right] \mathcal{U}\left[d_{2}-z-g(x)\right], & x \in\left(0, L_{1}\right) \\ \underline{\underline{\epsilon}}_{\mathrm{SNTF}}(z), & x \in\left(L_{1}, L\right)\end{cases}
$$

Other equations, results, and conclusions are not affected by this typographical error. All versions of the article were corrected on 12 March 2020.

*Address all correspondence to Akhlesh Lakhtakia, E-mail: akhlesh@psu.edu 